\title{
Problem Solving in Engineering Education and Professional Engineering Work
}

\section{Dr. Alexandra Vinson, Northwestern University}

Alexandra H. Vinson is a Postdoctoral Fellow in the School of Education and Social Policy at Northwestern University. She received her Ph.D. in Sociology \& Science Studies from the University of California, San Diego. Her research interests include professional education and enculturation in medicine and STEM fields.

\section{Dr. Pryce Davis, University of Nottingham}

Pryce Davis is an Assistant Professor of Learning Sciences at the University of Nottingham. He received his Ph.D. in Learning Sciences from Northwestern University. His research interests include learning in informal settings and public engagement with science.

\section{Prof. Reed Stevens, Northwestern University}

Reed Stevens is a Professor of Learning Sciences at Northwestern University. He holds a B.A. in Mathematics from Pomona College and $\mathrm{PhD}$ in Cognition and Development from the University of California, Berkeley. Professor Stevens began his professional career as a mathematics teacher. For the past two decades, he has studied STEM learning both in and out of school. His research seeks to understand how and when learning environments are productive for people and to translate those findings into practical use in the design and resdesign of learning environments. In recent years and in collaboration with colleagues at Northwestern, he created FUSE Studios to reimagine STEAM education around the values of innovation, making, and collaboration (https://www.fusestudio.net/). Since 2012, FUSE has been implemented in more than 135 school and community sites around the US and will soon begin in Helsinki, Finland. Stevens co-led two large National Science Foundation (NSF) research centers-the Center for the Advancement of Engineering Education (CAEE) and the Learning in Informal and Formal Environments Center (LIFE) — and has received numerous research grants from NSF and various private foundations. Articles of his relevant to the engineering education research community include "Becoming an engineer: Toward a three dimensional view of engineering learning" (2008) in the Journal of Engineering Education and "Professional engineering work" (2014) in the Cambridge handbook of engineering education research. 


\section{Problem Solving in Engineering Education \& Professional Engineering Work}

\section{Introduction}

Engineering is often described as science that is applied to solve real world problems. Indeed, problem solving is central element of engineering work (Sheppard, Colby, Macatangay \& Sullivan, 2006) and the ability to solve problems is a salient aspect of an engineer's identity (Anderson, Courter, McGlamery, Nathans-Kelly \& Nicometo, 2010). Problem solving ability is heavily emphasized in engineering education. However, engineering education has been criticized for emphasizing problems that may reflect students' ability to work with formulated and represented problems, but that do not resemble the types of problems students will encounter as professional engineers.

However, despite engineering educators' efforts to align the school and work contexts, scholars have noted that there is a scarcity of systematic analyses of engineering work (Trevelyan \& Till 2007, Stevens, Johri \& O’Connor 2013). Moreover, Trevelyan (2010) pointed out that engineering education operates on a model of engineering as design and technical problem solving, but that when this model is held up to actual engineering work, it turns out that engineering educators overlook the substantial role that socio-emotional and relational activities play in engineering work. Trevelyan thus identifies a mismatch in engineering education and work, ultimately concluding that engineering education curriculum change based on empirical studies of engineering work has the potential to strengthen engineering education. This conclusion is similar to the position of Stevens, Johri \& O'Connor (2013), who argue that the contexts of engineering education and professional engineering work should be understood in concrete detail and studied comparatively. They point out that there are not enough studies of everyday professional engineering work to understand the practices of engineering work and how they compare to the practices learned in engineering school.

One result of this lack of systematic attention to the work of early career engineers is that when engineering educators attempt to align the classroom with the workplace, they may give students an idea of engineering work that does not reflect what early career engineers will actually do in entry level positions (Jocuns, Stevens, Garrison \& Amos 2008). Following Stevens, Johri \& O'Connor (2013) and Trevelyan (2010; with Till, 2007), if we knew more about what entry level engineers actually do at work, engineering educators could use this to inform what they teach students in many areas, including problem solving. This could help align the communities of practice in school and work. Thus, one way to increase our understanding of engineering work practices is to watch new engineers as they learn to do professional engineering work.

To this end, our research team has carried out a study of the school-to-work transition of undergraduate engineering majors wherein we observe these early career engineers at work, often in their first jobs after graduation or in an internship. The primary contribution of our larger study is to provide systematic information of the normal working conditions of early career engineers. In this particular paper, we provide insight into the types of problems early career engineers encounter in the course of their work and how they solve them. 


\section{Data \& Methods}

This analysis is part of a National Science Foundation-funded study of the school-to-work transitions of early career engineers. An important contribution of our broader study is that we directly examine the transition from college to full-time work using a variety of qualitative methods, including ethnographic participant observation, interaction analysis of video data, and semi-structured interviews with college students, newly hired engineers, and more senior engineers. This stands in contrast to approaches that advocate for educational/curricular change based on assumptions about or relatively abstract conceptualizations of engineering education or of engineering work. Indeed, the notion that one must examine the work and professional identity of early career engineers directly is becoming more present in scholarly work (see Winters \& Matusovich, 2015; Trevelyan \& Till, 2007; Trevelyan, 2010; Stevens, Johri \& O'Connor, 2013). Many of these authors point out that direct observation of engineering work also highlights the importance of socio-emotional skills, such as leadership and teamwork skills, in professional engineering work (see also Baytiyeh, 2010; Anderson, Courter, McGlamery, Nathans-Kelly \& Nicometo, 2010).

In this paper we draw on interviews and workplace observations of 20 engineers to demonstrate the types of problems entry level engineers typically encounter in the workplace. During 2015 and 2016 we conducted observations of typical work activities at five different professional engineering settings: a steel mill, a supply chain management company, a company specializing in the design and sale of elevator systems, a third party medical device testing company, and a small firm specializing in device development for containerless processing. We also conducted monthly interviews with several participants who we were not able to observe at work, due to employer or security constraints. Early career engineers are those who are moving into the engineering workforce after completing their Bachelor's or Master's degree, as well as students who are working during school as interns or co-op students. The engineers we studied worked in engineering roles ('engineer' was in their job title) in a variety of different environments in these companies, including engineering consulting, manufacturing, continuous improvement, quality control, research and development, third party testing, and corporate management. Accordingly, these engineers come from various disciplinary backgrounds and universities. All names of persons and companies are pseudonyms. See Table 1 for further details.

During workplace observations, instances of problem solving activity were recorded through ethnographic field notes or through audio and video recording. These recording modalities were applied in the context of participant observation, during which we accompanied the engineers through their daily routines, supplemented with periodic semi-structured and field interviews with participants. The length of engagement with each engineer varied, as some underwent employment changes during the study. Length of engagement was also determined by how quickly we were able to achieve saturation, a sense of having seen the full range of their work activities.

\section{Findings}

Problem solving is one attribute of engineering work that engineers describe as particularly characteristic of their profession. For example, Chris, an early career engineer, described the centrality of problem solving in this way: 
About engineering particularly, what interests me is how to, I guess, there's always this need for people who have, I guess the training, and the kind of skill set to solve and tackle those really difficult problems. I like to think that I'm a problem solver, that people give me a task or a problem, and I try to knock it down, and take care of it, solve the problem. It's not so much, yeah. That's what really interests me about engineering. There are this group of people, engineers, who if you had a really difficult problem, or kind of thing you're struggling with, you would more likely go to an engineer because they have the kind of skill set and thinking mindset to handle these problems.

In this paper, we examine problem solving in everyday engineering work, paying attention to obstacles that arise during problem solving and how early career engineers resolve them. This is a way of getting at what happens between the time that the engineer is given a task and when $\mathrm{s} /$ he completes the task. In everyday work tasks, new information pops up during the process, resources must be assembled, expertise acquired, and so on. We conceptualize engineering problem solving as involving obstacles in order to ask: what types of obstacles trip early career engineers up? Moreover, when obstacles arise that divert or obstruct the routine course of work, how do early career engineers respond? Watching the disruption of work can clue us into what types of knowledge, skills, experience, materials and serendipity it takes to accomplish everyday work. Disruptions in work can show researchers what engineers must learn in order to accomplish professional engineering work. If work flows smoothly, it is easier to infer that the activity an engineer is carrying out is already known.

Our work is motivated by the overall lack of ethnographic studies of the early career engineer's work activity, as well as a scholarly consensus that there is a mismatch between what engineering schools teach and what engineering workplaces require. Characterizing the types of obstacles early career engineers encounter and the types of resolutions (Lave 1988, p. 139) they devise provides engineering educators with insight into how engineers use what they learned in school in everyday work situations, and may also lead to an understanding of the fundamental differences between workplace and school contexts and how engineering educators can tailor curricula to bridge this gap. We identify three types of obstacles: developmental obstacles, materials obstacles, and interpersonal obstacles. We define and elaborate these types of obstacles below. We also identify and illustrate types of strategies that early career engineers implement to resolve these obstacles.

\section{Common Obstacles \& Resolutions in Early Career Engineering}

The workplace problems of the early career engineer are a subset of engineering problems. It is difficult to precisely define this set, but in practice, early career engineers are given tasks by their managers (e.g. find and compile data, design a small object, optimize or troubleshoot a process that is already in place, repair a piece of code, modify an existing object to meet new standards). Moreover, early career engineers often do not make the final decision on anything, although their input is solicited and they provide reasoned recommendations to their managers, who support the development of their professional judgment (Goodwin 1994, Stevens \& Hall 1998). Based on our preliminary findings, we identified three types of obstacles: developmental obstacles, materials obstacles, and interpersonal obstacles. We also identified four common resolutions: expert consultation, resource consultation, trial and error, and transposing the problem into a familiar modality. We illustrate these obstacles and resolutions below. 


\section{Developmental Obstacles}

Developmental obstacles are similar to Jonassen \& Hung's (2008) conceptualization of internal factors that influence problem difficulty, identifying domain knowledge, experience, judgment and reasoning as factors that influence the course of work when engineers encounter obstacles. We call these obstacles developmental obstacles because they reflect a knowledge gap (including familiarity with software packages, research techniques, and design skills) or an experience gap (inability to determine whether one has solved a problem, or difficulty dealing with anomalous results). These obstacles are 'developmental' in the sense that they are resolved as the early career engineer learns through experience in the workplace. These types of obstacles can show areas of mismatch by exposing things not learned earlier that need to be learned now.

Many engineering students learn MatLab for basic coding and one drafting program (such as SolidWorks or AutoCAD). Some engineering students, particularly industrial engineers, learn Excel. However, we found that early career engineers often noted that they wished they had more training in computer-aided engineering drawing. A similar finding was noted in Hall \& Stevens (1994), suggesting that this is a long-standing lacuna in engineering education. When engineers in our study experienced work disruptions because they lacked software familiarity, the main resolution they devised was consulting external resources, most commonly via googling the problem and reading or watching a tutorial. We observed engineers using this method to learn how to use Excel, SolidWorks, and CREO. However, we also observed engineers using a different method to resolve software obstacles. Rajesh, who was unfamiliar with the CREO drawing program, consulted many online video tutorials and message boards in his attempt to figure out how to transform a 3-dimensional shape to meet new state code specifications for light fixtures. After he was not able to figure out how to widen a set of triangular and trapezoidal shapes using the computer program, he decided to work out the unknown sides of each polygon by hand using trigonometry. His way of addressing this obstacle was to re-present the problem into a more familiar modality (his undergraduate education had emphasized pen and paper mathematics); this resolution co-existed with his other resolution of consulting external resources such as video tutorials.

In addition to software familiarity, another developmental obstacle to completing engineering tasks is engineers' level of experience and professional judgment. This often became obvious when procedures were underdetermined by the affordances of the materials engineers were working with or when engineers were unsure if they had correctly followed a test procedure. For example, in his work at a Medical Device Testing Company, Liam was responsible for carrying out testing on medical devices and certifying those tests so that the devices themselves could gain regulatory permissions for sale and use. One day at work, Liam, Trevor and Cassandra were working together to carry out degradation testing on the logo of a small plastic device. In order to test the pigment that had been stamped onto the plastic, Liam asked Trevor and Cassandra to rub the logo with a bleach wipe to simulate cleaning during normal use. Cassandra asked, "So, for how much time do we do this?" Liam replied, "Uhh, I think, just clean it as if - there's no instructions for it, so just clean it as if ((pause)) you wanted it to be clean."

Later, Cassandra, Trevor and Liam worked to determine if an instance of degradation was caused by the bleach or during the stamping process. Liam was unable to determine if the defect was 
something he overlooked in the quality control process (a precursor to the bleach testing). Liam explained how he planned to document the irregularity on his paperwork, and Cassandra, who identified the defect, seemed satisfied with his strategy. But Liam noted that he wanted to discuss the notation of the defect with Jordan, a more senior lab member. After several minutes, Liam went to find Jordan and brought him to the lab. Liam explained the situation to Jordan:

We're trying to figure out, like, the more you stare at these, the more you can pick out irregularities. And these are units that I picked out because they had--wait, not 2 . Where did you come from? Oh, no, I did see that one, ok. These units, I picked them out because I spotted an irregularity I guess quickly [makes air quote gesture], I'm not sure how to define quickly, but they're units that I kind of, that caught my attention more than the other units. However, now we're kind of getting into a debate of where do we draw the line of what is degradation. Because if you look at some units long enough, it's like, well, there's this little speckle here, this little speckle there, um.

In this quotation, Liam is recounting his thought process to Jordan as he prepares to ask Jordan how he should document instances of irregularities in the ink of the logo that was stamped onto the plastic device. Liam does not know whether the irregularities stem from the manufacturing process or because the logo was wiped off with a bleach wipe, which would have to be noted as degradation in the test results. Jordan looked at the examples and then referred to the requirement in the protocol. Liam pointed out that there was not a formal requirement in the protocol, but Jordan asked Liam to pull out the sheet with the different cleaning requirements. Jordan listened to Liam's explanation of the situation and then began giving recommendations for how to address the situation:

The intent, basically, with labeling is that once it gets to the customer and someone uses it, it should remain legible. And so, the defects are, you know, a little--like, scratches or extra pad printing like outside of what the little [inaudible] to me does not render the label illegible. So, it's fine, but I would still note it and just note like, if it looks like for the, particularly the one where it looks like it just dripped past the edge or something like that--which is to be expected a little bit with pad printing and also with this material, like, pad printing on here is more likely to scratch and scar just because of the--and the finishes will, too--but that's one of the things that we already kind of knew about and that's what we discussed with the customer.

Importantly, during this expert consultation, Liam gained more information; for example, Jordan told Liam that he and the customer were already aware that this was a common problem with pad printed logos. Jordan continued to advise Liam by orienting him to the protocol, telling him to make notations about the condition of the logo when the protocol required it, and reassuring him that they would be able to capture the details of the degradation in the report and discuss it with the customer. This is an example of a developmental obstacle because it resulted from gaps in knowledge and experience, but was corrected through consultation with a local expert. By consulting an expert, Liam, Trevor and Cassandra acquired additional information about the context that their task was situated in, as well as tailored guidance for completing the task. This helped them overcome the indeterminacy that they perceived in the protocol and helped them distinguish meaningful defects from non-meaningful defects. In a work context where such 
interactions are frequent, early career engineers gain the knowledge and experience they need to carry out their work, gradually developing their professional judgment and growing more independent.

\section{Materials Obstacles}

The second type of obstacle we discovered were materials obstacles. Materials obstacles are not unique to early career engineers - they can happen to anyone. One of the important insights of research in Science and Technology Studies, particularly in the work of Latour and Callon (Callon 1984, Johnson [Latour] 1988), was to show how material objects participate in scientific knowledge creation or social life, often through resisting or redirecting human effort. This insight laid the groundwork for a body of work on how the material world plays an important role in social and scientific life, causing theorists to look beyond human actors to include 'nonhumans' in their analyses. The material world can push back on humans' efforts to get work done, and engineers of all levels can face obstacles in assembling the material resources necessary to complete a task, or getting those material resources to cooperate.

Materials obstacles delay the course of work, sometimes significantly. During a series of tests on a handheld medical device, Liam explained that in order to move on to the next phase of testing, a batch of devices needed to be acclimated at a constant temperature. Liam planned to carry this out over the weekend, but the $\mathrm{A} / \mathrm{C}$ in the office was not always reliable. He told Davis, a member of our research team, that he needed to go up on the roof to change the air filter, with the hope that this would prevent the $\mathrm{A} / \mathrm{C}$ from malfunctioning and disrupting the acclimation process. If the acclimation were to fail, Liam would not be able to move on to the next phase of testing on Monday.

Engineers also experience difficulty finding the materials they need to build or assemble machinery, and can devote significant amounts of time to resource hunting. In one example, Walter was building a table to hold a mounted camera, and he was trying to build as much of it from scrap materials as possible. Walter investigated the scrap pile to see if any of the pieces met the specifications, but ultimately concluded that he would need to order the pieces from a supplier.

In another case, Priya was asked to resolve a process error in the company's ordering process. She had created a test order to see if all of the proper components would be shipped in the package. Because her company relies on having all of the proper materials shipped to remote job sites for installation, the orders need to be right the first time. When she discovered the error, she devoted most of her work week to assembling a list of all of the local and remote experts involved in the ordering and shipping process, as well as finding the parts that had been shipped in the test order. These parts were in a different office building about five minutes away; the building was an active construction site and she had to find one specific person to figure out where the parts were and another person to help her get the heavy boxes off of a tall warehouse shelf. Once she had the individual parts, she had to assemble the system to resemble how a customer might use it. She needed to find out if the proper software was installed onto the server. However, because the server was shipped with European plugs instead of American plugs, Priya had the additional obstacle of finding a computer power cord that was not currently in use (she ended up disassembling another computer system that was not in use). Due to the complexity of 
assembling all of the materials she needed in order to identify the source of the process error, this ended up taking up many of Priya's work hours that week.

In her case, Priya used a combination of expert consultation and trial and error to resolve the materials obstacles she encountered while trying to complete her work task: identifying the source of the process error and overseeing the correction of the ordering and shipping system on the developer side. Specifically, the materials obstacles were finding the actual parts that had arrived in the shipment and acquiring extra parts to be able to assemble and test the system. Consulting local experts also helped Priya overcome some developmental obstacles she faced, such as not knowing how to work the server system (corrected through didactic training with the on-site expert). She also worked with a more experienced engineer to learn about the development end of the ordering process.

\section{Interpersonal Obstacles}

Another type of obstacle we found during problem solving is interpersonal. Interpersonal obstacles describe situations where engineers need to assemble resources, consult experts, and grow professionally. Engineers who ask themselves, "how do I get what I need to do my work?" or "how do I get senior colleagues to invest in my development?" are encountering and addressing interpersonal obstacles. In this way, interpersonal obstacles and developmental obstacles are related, because when engineers identify something they need to learn, they also have to get someone to invest in helping them learn it. The individual interpersonal skills of the early career engineer can also cause or ease obstacles in the workplace; for example, an early career engineer who is difficult to get along with, very shy, or skilled at forging relationships with others. While interpersonal obstacles are not always related to discrete work tasks, an engineer's social skills can influence how tasks are carried out.

An engineer who is particularly well connected socially can have an easier time gathering information to complete a work task, particularly if s/he needs to gather backchannel information or rely on colleagues' candor. In one example, Hannah, a member of the Quality Team at a steel mill, needed to find out why a slab of steel was being transported back and forth between two storage locations, especially since she had submitted written documentation weeks before to have it taken to the scrapyard. One constraint in her situation was that if she used the easiest method for resolving the transport error, her department would be charged for the transportsomething she hoped to avoid, since charges were expensive and her department was not at fault. When she called one of the people in the transport department, he told her that the transporters do not read the transport slips. This was a moment of candor that resulted from Hannah's social rapport with other mill employees, and it helped her pinpoint the part of the process that was resulting in the transport error.

A few minutes later, she managed to catch a transport department supervisor as he was passing through the office. Hannah raised her concern that the transporters do not read the transport slips, explaining that it was a problem because she, as the originator of the transport slips, writes comments and detailed directions on the form. The supervisor became hostile, repeatedly stating that this was the only one they had messed up and downplaying her concerns until she backed off. While Hannah was able to use interpersonal skills to accomplish the work task of getting this 
individual slab scrapped, she faced an interpersonal obstacle when she tried to address the larger issue of the transporters not reading the transport slips.

In another working paper, our research team explores the issue of mentorship (Davis, Vinson \& Stevens 2017). The paper presents successful and unsuccessful interactions between early career engineers and more senior engineers, some of whom become mentors to these engineers. Taken together, our team's findings in this area point to the importance of moving beyond strictly cognitive conceptions of learning and reinforce the importance of engineering education initiatives that incorporate teamwork, clients and stakeholders into educational activities.

\section{Common Resolutions Devised by Early Career Engineers}

Although we discussed the resolutions early career engineers frequently devise in response to the examples of obstacles we gave above, we want to give a few more examples of resolutions and problem solving methods in this section.

First, we showed that when confronted with an obstacle that prevents them from completing a work task, early career engineers often consult local experts. These range from asking superiors to clarify instructions or asking colleagues for tips on procedure. For example, we commonly observed early career engineers asking colleagues for assistance with advanced Excel maneuvers. There are also encounters like the example of Liam consulting Jordan that was noted above; in that case, Jordan helped to structure the task for Liam by reorienting him to the experimental protocol. Jordan also provided additional context for the testing by telling Liam that the customer and the senior engineers were already aware of the problem he had identified. Expert consultation allows a more senior engineer to make judgment calls and final decisions, particularly in anomalous situations.

Similar to local expert consultation is consulting resources like online message boards, YouTube software tutorials, quality/materials standards (ASTM, ANSI, ISO), manuals, and protocols . Early career engineers were quick to consult these resources if they encountered an obstacle, and, in particular, many of them overcame their developmental obstacle of low software familiarity through this type of self-teaching.

A third resolution strategy we observed was trial and error, which frequently involved tinkering with hardware or software until the engineer figured out what the problem was and how to resolve it. We observed many hours of successful and unsuccessful tinkering. In one case, we observed Ryan as he built and conducted troubleshooting on a Programmable Logic Controller (PLC). Not only did he configure the hardware itself, he also had to learn the programming software. This was given to him by his mentor as a learning exercise, and he worked on it mostly in isolation. There were no readily available local experts for him to contact, and he had limited success in consulting external resources, although he mentioned being interested in taking a formal PLC course.

In a similar case, Priya was attempting to figure out why a display monitor was not showing a picture when it was plugged in. She carried out extensive troubleshooting using a multimeter in order to figure out if current was passing through the power supply, and she examined the components systematically to attempt to identify the source of the problem. After several hours 
of tinkering, she managed to track down a local expert who identified a missing piece of the system - the computer that generated the image to be displayed. With his help, she was able to set the system up and verify that it was functional. In Priya's case, her method of tinkering revealed a structured approach to testing the system that may have reflected aspects of her engineering education:

Vinson: When you have a system that might be broken in some way do you have a particular way that you go about figuring out what's wrong?

Priya: $\quad$ Yeah, whenever we know that it should work and it's not working, we start looking at circuit point by point. That is called debugging a circuit.

Finally, sometimes if engineers encounter an obstacle during problem solving, they re-present the problem into a more familiar modality, also theorized as finding a workaround (Alter 2014). We observed this with software familiarity. In the section on developmental obstacles we mentioned that Rajesh resolved a software familiarity obstacle by working out a trigonometry problem by hand to find lengths of polygon sides for a fixture he was designing. We also observed engineers who sketched a design out by hand before drawing it with drafting software. When Walter, mentioned above, was building his camera mount, he drew a rough sketch on paper to get an idea of what he wanted to build. We did not observe problem re-presentation as often as other resolution methods, but we note it as one way that engineers use existing competencies while they are still learning new workplace skills.

\section{Discussion \& Conclusion}

In this paper, we discussed the types of obstacles that keep early career engineers from completing their work tasks. Through observation and interviews, we show what types of resolutions engineers devise to overcome these obstacles. Our findings have the potential to inform engineering educators as they continually adapt their curricula to prepare their students to succeed in the workplace. One motivation for carrying out this study is the commonly noted mismatch between what is taught in engineering education and what is required to succeed in the workplace. However, this study is also motivated by a desire to understand the organization of engineering work. Engineers, like other professionals, carry out work that can have broad consequences for society and the material world. However, engineers are also workers and are embedded in work organizations, schools and labor markets. Our approach allows us to study how new engineers become embedded in work organizations, how they are shaped by their education, and how they carry out their work. By examining how new engineers learn to do their work, we can better understand the relationship between the individual engineer and the context he or she is embedded in - and how this relationship changes over time.

Our findings indicate that, while early career engineers do encounter problems at work, they are able to resolve them using a number of different strategies. Notably, the resolutions themselves do not draw mainly or exclusively on the technical skills emphasized in engineering education, but rather on skills related to information acquisition, including the social skills needed to get help from more senior engineers. Their information-seeking skills also extended to online searching, which may be a solution method that they use in other areas of their life that also 
works in the engineering workplace. As they learn about field-specific resources like manuals, protocols and compilations of standards, they are also likely to reach for those, as well.

There is variation in the type of curricula offered by engineering schools, and in our sample we had a range of schools with different levels of emphasis on experiential learning. Those schools with less experiential learning courses tended to feature more courses where students learned concepts and demonstrated competence through traditional exercises like problem sets and exams. Engineering educators have steadily incorporated problem-based learning exercises, projects and capstone experiences into undergraduate engineering education. Problem-based Learning (PBL) has been one technique introduced in order to bring 'real life' problems into the classroom. Those educational exercises, particularly PBL, emphasize information-seeking as a skill that will be developed through participation in the exercise. What we may see in these data is the payoff of those activities, but as one respondent described, there are still meaningful differences between doing a school project and doing professional engineering work. During an interview, Karen suggested that one good question to ask her and other interviewees would be to discuss the things about professional engineering work that she wished she had known as an undergraduate:

Karen: $\quad$ Okay. I guess if I were to ask a question, it would be, "what has been the biggest difference that you've learned or realized in working that you wish you had known in undergrad?"

Vinson: $\quad$ Yeah. What is that for you?

Karen: $\quad$ We did a lot of projects. I had a senior design project, I had a project measure expectations class [inaudible 00:52:59], where we worked in groups and would work on a real, already done project, but starting it for ourselves and going through that project. We worked on a lot of things, but definitely working on a project in my civil engineering projects was definitely different than working on a project in the real world. Even when I was working on projects in my classes, it was very much a college setting where you would get together at one big table at the library and work on things together, whereas that's not the type of studying that you have a project in the real world. Better understanding what was expecting or what was actually done in the really world might have been nice.

Academically, they prepared me to an extent, you can't learn everything, but environmentally preparing you for how the structure of design, or what sectors in design there were, or even construction within that field, I was not ... [prepared].

Karen was able to identify areas of professional engineering work that might seem similar (working on projects), but also to specifically say how 'working on a project' in engineering school is qualitatively different from 'working on a project' in a professional engineering setting. Using similar words to describe activity in different contexts can actually hide differences in practice, reinforcing the importance of directly examining engineers as they do their work (see Stevens, O'Connor, Garrison, Jocuns \& Amos 2008 for a discussion of how the phenomenon of 'teamwork' can vary in meaningful ways across different contexts). The shortcomings of traditional engineering education and PBL can be offset with work experience; however, Karen 
had limited industry experience as an undergraduate (only one term of internship). At the end of a discussion about reading graphs to interpret the quality of a pour of molten steel, Gwen described how work experience in industry helped her learn different things than she would have in the classroom:

This is an example of what I'm learning [in my internship]. Because they don't teach us that in college. They teach us ideal. This is what would happen if everything goes perfectly. They don't mention what would happen when things get screwed up.

Engineering students have been described as 'overbuilt' from a perspective of technical knowledge, especially with respect to usable and needed technical knowledge, and 'underbuilt' on other aspects of engineering, such as the social aspects of a socio-technical education. In our study, engineers indicated that the work they did on a daily basis did not draw on many of the skills they demonstrated to receive their engineering degree, and required some skills that they had not developed during college. The clearest skill gap was in the area of software familiarity, with many students having experience using MatLab and one design program, but missing skills in Excel, AutoCAD, CREO, and/or SolidWorks needed to succeed at work.

However, engineers also demonstrated resourcefulness for dealing with obstacles that did arise. In general, we found that engineers were aware of the differences between school and work settings, and took advantage of learning opportunities as they arose. Particularly in the case of Gwen, although she was not resolving an obstacle, in the situation described above, she was having a conversation with a more senior engineer and learning from him about assessing steel quality. As she pointed out, this was not something she would be able to learn in a school setting, thereby reinforcing the value of early work experience as complementing what is learned in the classroom.

One pertinent question for future research is how much workplace preparation should engineering schools be held accountable for? While the specifics of every industry and work setting cannot be taught in engineering school, there is a middle ground where engineering educators try to make professional engineering work more familiar to students while they are still in school. This is happening in PBL and is aided by engineering education scholars who try to identify necessary competencies for workplace success. By pinpointing skills gaps, our research may identify opportunities for intervention in college that will smooth the post-graduation transition. However, as we have pointed out in other work (Vinson \& Stevens 2016), pregraduation work experience also allows early career engineers opportunities to discover the similarities and differences between engineering school and professional engineering work, even when it comes to learning problem-solving skills.

\section{Acknowledgements}

This research was supported by a grant from the National Science Foundation (\#1252372). We would like to thank two anonymous reviewers and the program chair for their comments on earlier versions of this draft. 
Table 1: Study Participants \& Field Sites

\begin{tabular}{|l|l|l|}
\hline \multicolumn{1}{|c|}{ NAME } & \multicolumn{1}{|c|}{ TYPE OF } & \\
\hline Karen & Civil & EnginEER \\
\hline Julia & Chemical & Large Petroleum Company \\
\hline Kyle & Chemical & Large Chemical Company \\
\hline Lydia & Civil & Engineering Consulting Firm 1, Engineering Consulting Firm 2 \\
\hline Peter & Industrial & General Consulting Firm \\
\hline Chris & Industrial & Current student--Intern at Supply Chain Management Company \\
\hline Margaret & Industrial & Supply Chain Management Company \\
\hline Sasha & Mechanical & Large Southern Steel Mill \\
\hline Rajesh & Electrical & International Elevator Company \\
\hline Leah & Electrical & Large Southern Steel Mill \\
\hline Steve & Computer & Current student--Intern at Dynamic Instruments Midwest \\
\hline Liam & Science & \\
\hline Gwen & Materials Science & Current student--Co-op at Large Southern Steel Mill \\
\hline Trevor & Biomedical & Current student--Intern at Medical Device Testing Company \\
\hline Cassandra & Biomedical & Current student--Intern at Medical Device Testing Company \\
\hline Curtis & Chemical & Large Southern Steel Mill \\
\hline Walter & Mechanical & Large Southern Steel Mill \\
\hline Hannah & Materials Science & Large Southern Steel Mill \\
\hline Priya & Electronics & International Elevator Company \\
\hline Ryan & Electrical & Large Southern Steel Mill \\
\hline
\end{tabular}

\section{References}

Alter, S. (2014). "Theory of Workarounds." Communications of the Association for Information Systems, 34:1041-1066.

Anderson, K., S. Courter, T. McGlamery, T. Nathans-Kelly \& C. Nicometo (2010).

"Understanding engineering work and identity: a cross-case analysis of engineers within six firms.” Engineering Studies, 2(3):153-174.

Baytiyeh, H. (2010). "Disparity between College Preparation and Career Demands for Graduating Engineers." International Journal of Engineering Eduction, 28(5):1221-1231.

Callon, M. (1984). "Some elements of a sociology of translation: domestication of the scallops and the fishermen of St Brieuc Bay." The Sociological Review, 32(S1):196-233.

Davis, P., A. H. Vinson \& R. Stevens (2017). "Informal Mentorship of New Engineers in the Workplace." Proceedings of the 2017 American Society for Engineering Education Annual Conference \& Exposition. 
Goodwin, C. (1994). "Professional Vision.” American Anthropologist, 96(3):606-633.

Hall, R. \& R. Stevens (1994). "Making space: a comparison of mathematical work in school and professional design practices." The Sociological Review Monograph Series: The Cultures of Computing, Susan Leigh Star, Ed., 42(S1):118-145.

Jocuns, A., R. Stevens, L. Garrison \& D. Amos (2008). "Students' changing images of engineering and engineers." Proceedings of the 2008 American Society for Engineering Education Annual Conference and Exposition.

Johnson, J. (1988). "Mixing Humans and Nonhumans Together: The Sociology of a DoorCloser." Social Problems, 35(3).

Johri, A. \& B.M. Olds (2011). "Situated Engineering Learning: Bridging Engineering Education Research and the Learning Sciences.” Journal of Engineering Education, 100(1):151-185.

Jonassen, D. \& W. Hung (2008). "All Problems are Not Equal: Implications for Problem-Based Learning." Interdisciplinary Journal of Problem-Based Learning, 2(2):6-28.

Lave, J. (1988). Cognition in Practice: Mind, Mathematics and Culture in Everyday Life. Cambridge: Cambridge University Press.

Sheppard, S., A. Colby, K. Macatangay \& W. Sullivan (2006). "What is Engineering Practice?" International Journal of Engineering Education, 22(3):429-438.

Stevens, R, K. O’Connor, L. Garrison, A. Jocuns \& D. Amos (2008). “Becoming an Engineer: Toward a Three Dimensional View of Engineering Learning." Journal of Engineering Education, 97(3):355-368.

Stevens, R., A. Johri \& K. O’Connor (2013). "Professional Engineering Work.” In A. Johri \& B.M. Olds (Eds.), Cambridge Handbook of Engineering Education Research. Cambridge: Cambridge University Press.

Stevens, R. \& R. Hall (1998). "Disciplined perception: Learning to see in technoscience." In M. Lampert \& M. L. Blunk (Eds.), Talking mathematics in school: Studies of teaching and learning (pp. 107-149). Cambridge: Cambridge University Press.

Trevelyan, J. \& S. Till (2007). "Published Research on Engineering Work." Journal of Professional Issues in Engineering Education And Practice, 133(4):300-307.

Trevelyan, J. (2010). "Mind the Gaps: Engineering Education and Practice." Proceedings of the 2010 AaeE Conference, Sydney, Australia.

Vinson, Alexandra H. \& Reed Stevens (2016). "Staying in or Getting Out: The Relationship Between Undergraduate Work Exposure and Job Satisfaction after Graduation." Proceedings of the 2016 American Society for Engineering Education Annual Conference \& Exposition. 
Winters, K. \& H. Matusovich (2015). "Career Goals and Actions of Early Career Engineering Graduates." International Journal of Engineering Education, 31(5):1226-1238. 